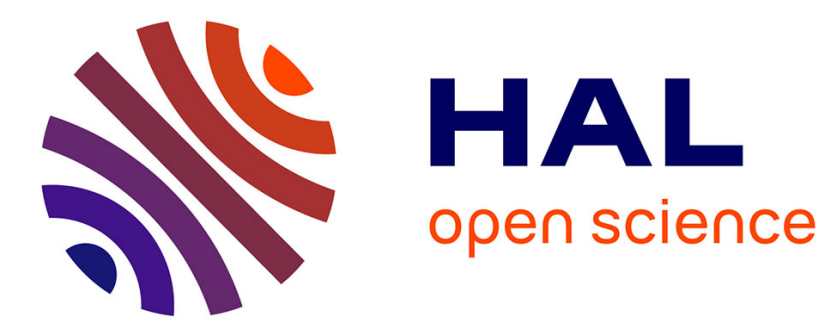

\title{
Non-invasive Myocardial Shear Wave Elastography Device for Clinical Applications in Cardiology
}

Mafalda Correia, Ilya Podetti, Olivier Villemain, Jérome Baranger, Mickael Tanter, Mathieu Pernot

\section{- To cite this version:}

Mafalda Correia, Ilya Podetti, Olivier Villemain, Jérome Baranger, Mickael Tanter, et al.. Noninvasive Myocardial Shear Wave Elastography Device for Clinical Applications in Cardiology. Innovation and Research in BioMedical engineering, 2017, 38 (6), pp.357-362. 10.1016/j.irbm.2017.09.001 . hal-01682319

\section{HAL Id: hal-01682319 \\ https: / hal.sorbonne-universite.fr/hal-01682319}

Submitted on 12 Jan 2018

HAL is a multi-disciplinary open access archive for the deposit and dissemination of scientific research documents, whether they are published or not. The documents may come from teaching and research institutions in France or abroad, or from public or private research centers.
L'archive ouverte pluridisciplinaire HAL, est destinée au dépôt et à la diffusion de documents scientifiques de niveau recherche, publiés ou non, émanant des établissements d'enseignement et de recherche français ou étrangers, des laboratoires publics ou privés. 


\section{Non-invasive Myocardial Shear Wave Elastography device for clinical applications in cardiology}

Mafalda Correia ${ }^{1}$, Ilya Podetti ${ }^{1}$, Olivier Villemain ${ }^{1}$, Jerome Baranger ${ }^{1}$, Mickael Tanter $^{1}$ and Mathieu Pernot $^{1}$

${ }^{1}$ Institut Langevin, ESPCI Paris, PSL Research University, CNRS UMR7587, INSERM U979, Paris 6 University and Paris 7 University

17, Rue Moreau

75012 Paris

France 


\section{Abstract}

Background: Ultrasound Shear Wave Elastography has been widely used in clinical practice to access tissues' stiffness non-invasively. However, the application of this technique to access myocardial stiffness clinically and non-invasively was yet not demonstrated. In this study, we introduce a new prototype for clinical application purposes, the Myocardial Shear Wave Elastography imaging (MSWEi) device.

Methods: The MSWEi device lays on a linear phased-array probe (2.75-MHz, Vermon S.A., Tours, France) connected to an ultrafast ultrasound scanner (Aixplorer, Supersonic Imagine, Aix-en-Provence, France), a dedicated sequence of Shear Wave Elastography and unfocused emissions at very high frame rate for myocardial stiffness evaluation, and a dedicated graphical user interface for physicians use in clinical settings.

Results: This prototype was evaluated and validated in-vitro using calibrated mimicking tissue phantoms, providing accurate and robust measurements for tissues' stiffness up to $25 \mathrm{kPa}$. The device was also validated for stiffness estimation on different thin layers for thickness superior to $5-\mathrm{mm}$, showing a bias estimation inferior to $15 \%$. Finally, the in-vivo and non-invasive application of the prototype was also evaluated on a patient.

Conclusion: This study presented a new device to evaluate non-invasively myocardial stiffness using an ultrafast ultrasound scanner in a clinical setting. The in-vivo, non-invasive and clinical feasibility was demonstrated showing the potential of the device to evaluate myocardial stiffness accurately up to $25 \mathrm{KPa}$ and for myocardial wall thickness superior to 5-mm.

\section{Introduction}

In the past decades, Elastography techniques, based on ultrasound imaging, have been introduced to assess the elastic properties of soft tissues non-invasively (consult Gennisson et al. 2013 for a review of the principles and techniques). Shear Wave Elastography (SWE) imaging techniques are based on mechanical shear waves propagation imaging in the tissue (propagation velocities typically varies between 1 and $10 \mathrm{~m} / \mathrm{s}$ ) induced through the application of time varying external forces. One of these SWE techniques, presented by (Bercoff et al. 2004), consists in (1) the generation of a push-beam within the tissue of interest using acoustic radiation force, (2) imaging the resulting shear-wave propagation at ultrafast frame rate and (3) estimating the local velocity of the shear wave propagation, which can be used 
to determine the local stiffness of tissues varying complexities, e.g., the cardiac muscle, i.e. the myocardium.

The myocardium is a complex three-dimensional network of particularly organized, specific and elastic fibers with physical properties that change in time and spatially. The evaluation of the myocardial stiffness could help the diagnosis of several conditions allied to stiffness changing, e.g. heart failure. Different studies have shown the feasibility of quantifying myocardial stiffness in-vivo in open chest animals (Hsu et al. 2007; Pislaru et al. 2009; Pernot et al. 2011; Bouchard et al. 2011; Pernot et al. 2016). Recent studies have demonstrated that non-invasive transthoracic myocardial stiffness measurements is feasible in few normal subjects at high temporal resolution (Song et al. 2013; Papadacci et al. 2014; Correia et al. 2016). However, myocardial stiffness measured non-invasively in clinical practice on patients with cardiac disease was yet not demonstrated. In this study, we introduce a clinical implementation of Myocardial Shear Wave Elastography using ultrafast imaging at very high frame rates, a device for clinical evaluation purposes. Furthermore, we validate this implementation on in-vitro calibrated isotropic phantoms which mimic the myocardial thickness and stiffness.

\section{Material and Methods}

\section{Myocardial Shear Wave Elastography Imaging (MSWEi) device for clinical purposes}

A 2.75-MHz linear phased-array probe (96 elements, 0.2-mm pitch, Vermon S.A., Tours, France) connected to an ultrafast ultrasound scanner (Aixplorer, Supersonic Imagine, Aix-en-Provence, France) was used to perform the acquisitions. An electrocardiograph (ECG) system (AccuSync 42, AccuSync Medical Research Corporatio, CT, USA) was used to trigger and measured ECG signals in real time, which was recorded by a data acquisition board (USB-201 model, MC Measurement Computing, MA, USA). A dedicated software with a graphical user interface (Matlab 2015a, MathWorks, Natick, MA, USA) was developed for this application and used on a tablet (Surface Pro 3, Microsoft Corporation, Redmond, Washington, USA), Figure 1, to run and perform the acquisitions.

The imaging sequences were designed to 1 ) perform a primary ultrafast harmonic B-mode image (Correia et al. 2016) followed by 2) Shear Wave Elastography acquisition (Papadacci et al. 2014; Correia et al. 2016). The imaging sequences developed and used in this study were based on the emission of unfocused ultrasound beams, i.e., diverging-waves. For details on diverging-waves transmission concept and formation, see (Papadacci et al. 2014). 


\subsection{B-mode and Myocardial Shear Wave Elastography Imaging}

Prior to Myocardial Shear Wave Elastography, a B-mode image was acquired for anatomical landmark purposes. This B-mode image was based on Ultrafast Harmonic Coherent Compound Imaging, presented by (Correia et al. 2016), using 15 diverging-waves transmission at 2.1-MHz.

After B-mode imaging, Shear Wave Elastography was launched with ECG-trigger synchronization. To induce a shear wave propagation within the tissue, a push-beam, through acoustic radiation force, was transmitted at 3-MHz, 300- $\mu$ s duration and at a push-depth of interest. Immediately after generating the shear wave, ultrafast imaging was launched at frame rates superior to 1000 images/s. Ultrafast imaging sequence consisted on the successive transmission of 2 diverging-waves at 3-MHz (Papadacci et al. 2014). The acoustic output of Myocardial Shear Wave Elastography imaging sequences were measured using a calibrated interferometer in water. The sequences fulfilled the Food and Drug Administration (FDA) requirements (510k track 3, FDA) defined by the mechanical index (MI) and the spatial-peak time average (I IPTA $)$ with a derating factor of $0.3 \mathrm{~dB} \mathrm{~cm}^{-1} \mathrm{MHz}^{-1}$. The $\mathrm{Ml}_{0.3}$ was set to $1.8\left(\mathrm{MI}_{0.3}<1.9\right.$ by FDA) and the ISPTA was set to $157 \mathrm{~mW} \mathrm{~cm}^{-2}$ (ISPTA $0.3<720 \mathrm{~mW} \mathrm{~cm}^{-2}$ by FDA), with a repetition time of 1 second.

In what concerns in-vivo human imaging experimentation, informed consents were obtained within the clinical investigation protocol $\mathrm{n}^{\circ}$ 2015-A00187-42 approved by the CPP (Comité de Protection des Personnes), lle de France VI, France.

\subsection{Graphical User Interface}

The graphical user interface was developed with the purpose of allowing complete independence between the physician and the ultrafast ultrasound scanner and perform Shear Wave Elastography for myocardial stiffness measurements application. Parameters as the main information of the patient in study, the choice of the push focusing depth and ECG trigger timing within the cardiac cycle were integrated, as it is showed in Figure 1. 


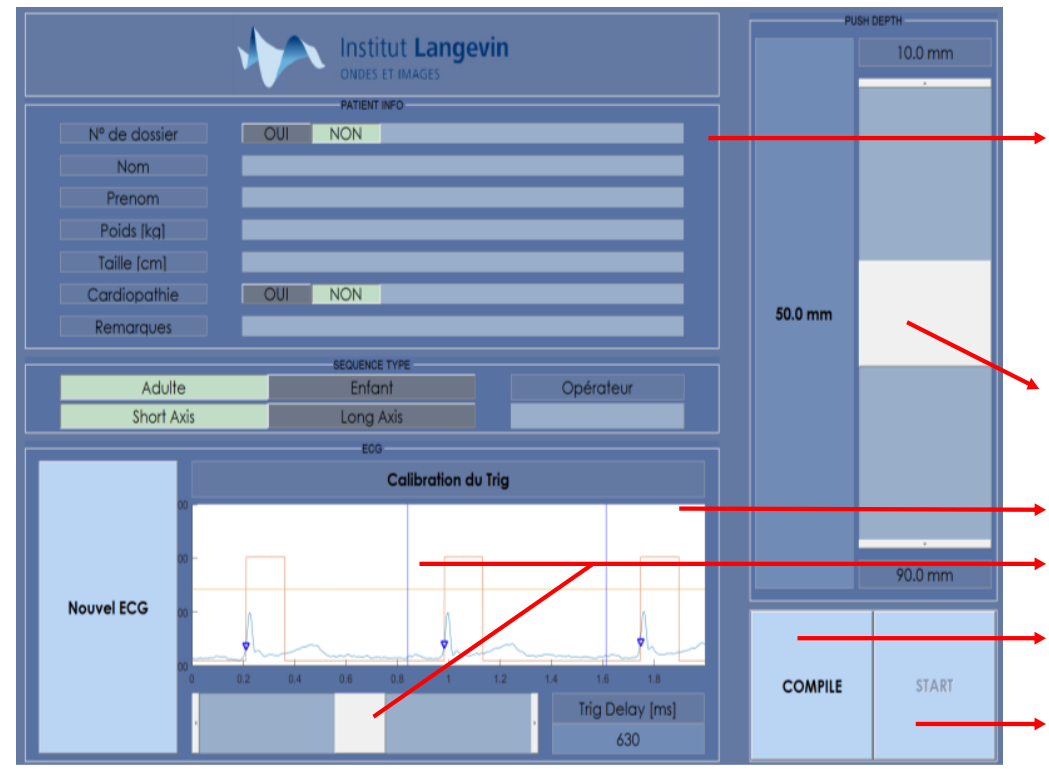

Patient Information

Reference number

Last Name

First Name

Weight

Size

Cardiomyopathy

Notes

Push Depth slider

Real time ECG visualization

ECG trigger choice

Compile Parameters of the sequence (ECG trigger, Push depth, Patient's Info)

FIGURE 1 : GRAPHICAL USER INTERFACE FOR CLINICAL MYOCARDIAL SHEAR WAVE ELASTOGRAPHY IMAGING.

\subsection{Post-processing analysis: shear wave propagation velocity measurements}

The radio-frequency (RF) signals of the acquisition were first backup in the tablet. The RF data were processed off-line with a Matlab (2015a, The MathWorks Inc., Natick, MA, USA) interface. The RF-data were then reconstructed offline using a conventional delay-and-sum beamforming algorithm, implemented in a graphical processing unit (K600, NVidia, Santa Clara, CA, USA).

Tissue velocity images were attained using 1-D cross-correlation of successive beamformed images with cosine interpolation (1.5-mm kernel size and 97.5-\% overlap), (Bonnefous et al. 1986; Sandrin et al. 1999; McLaughlin \& Renzi 2006), followed by scan-conversion. Subsequently, spatiotemporal tissue velocity data were computed and shear wave propagation velocities in the lateral direction were calculated using linear least-squares estimation on temporal 1-D cross-correlation with different lateral spatial lags (lags range: 0.2 to 1.8-mm) (McLaughlin \& Renzi 2006; Correia et al. 2016). Finally, the shear modulus ( $\mu$ ) can be calculated by the followed relationship (Equation 1):

$$
\text { Equation 1: } \quad \mu=v_{s}^{2} \rho
$$

where the $v_{s}$ corresponds to the shear wave propagation velocity and $\rho$ the tissue's density. The shear modulus is linked to a measure of stiffness, through the Hooke's law.

\section{Evaluation and validation on in-vitro calibrated phantoms}

Isotropic Polyvinyl alcohol (PVA) hydrogels have been largely used as tissue mimicking models for Shear Wave Elastography imaging due to their elastic mechanical properties. In this study, we investigated the 
performance of our device to provide accurate stiffness on various PVA hydrogels of different thickness and different stiffness. First, we developed soft isotropic models with different thicknesses to mimic the in-vivo anatomical differences of the myocardial thickness (e.g. normal against hypertrophic or dilated myocardium), and soft isotropic models with different stiffness to evaluate and validate the limits of our technique to assess myocardial stiffness.

In the first model configuration, eight different samples were created and constituted by $10-\%$ PVA solution (molecular weight 89 000-98 000, 99+\% hydrolyzed, Sigma-Aldrich, St Louis, US), 1-\% cellulose (20 $\mu \mathrm{m}$ in diameter, S3504 Sigmacell, Sigma-Aldrich, St Louis, US) and 89-\% distilled water. Samples were transferred into a parallelepiped PVC mold ( $80 \times 50 \times 50-\mathrm{mm} 3)$ and underwent two isotropic freezing/unfreezing cycles (i.e. approximately $20^{\circ} \mathrm{C}$ during $12-\mathrm{h}$ followed by $-18^{\circ} \mathrm{C}$ during $12-\mathrm{h}$ ). Then, the samples were cut in slices with different thickness, i.e. from 1.8 to $18 \mathrm{~mm}$ thick (Figure 2). The purpose of this experimental configuration was to evaluate the tissue thickness effect under the same tissue stiffness.

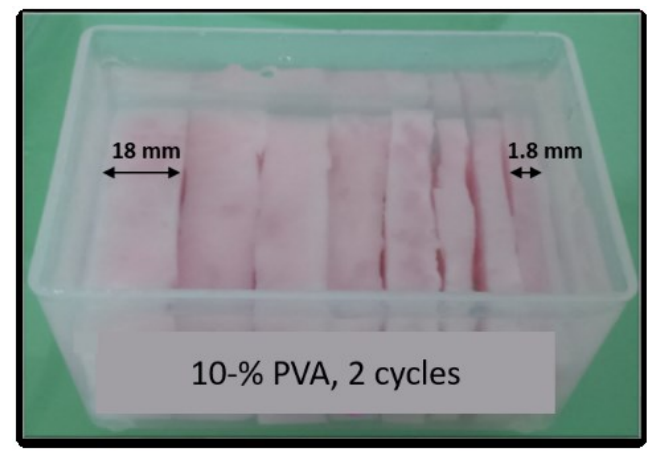

FIGURE 2: THE FIRST SOFT ISOTROPIC MODEL CONFIGURATION. EIGHT DIFFERENT SAMPLES OF DIFFERENT TICKNESSES (FROM 1.8 то 13 мМ тнІСК).

In the second model configuration, twenty different samples were created and they were divided equally in five groups of different PVA percentages, i.e. different media stiffness. Therefore, they were divided in: (1) 5-\% PVA solution, 1-\% cellulose and 94-\% distilled water; (2) 7.5-\% PVA solution, 1-\% cellulose and 91.5\% distilled water; (3) 10-\% PVA solution, 1-\% cellulose and 89-\% distilled water; (4) 12.5-\% PVA solution, 1-\% cellulose and $86.5-\%$ distilled water; and (5) 15-\% PVA solution, 1-\% cellulose and $84-\%$ distilled water. All samples were transferred into a parallelepiped PVC mold ( $80 \times 50 \times 50-\mathrm{mm} 3)$. For each group, each sample underwent 2 to 5 isotropic freezing/unfreezing cycles (i.e. approximately $20^{\circ} \mathrm{C}$ during 12 -h followed by $-18^{\circ} \mathrm{C}$ during $12-\mathrm{h}$ ) to induce elastic property changings, e.g. media stiffness changings.

For all configurations, the experimental setup included an absorber (Polyurethane, NPL, UK) covered by the PVA samples, immersed in water to guarantee good acoustic coupling. The experimental setup is presented in Figure 3 with the phased-array probe positioned at 5-cm distance of each sample. 
To evaluate the results given by the MSWEi device imaging, as presented in the last section, four acquisitions were performed for each PVA sample for reproducibility purposes and they were compared with the Aixplorer ultrasound scanner, using the clinical system and a 10-2-MHz linear-array probe, which was considered as the gold-standard measurements. A comparison in function of tissue stiffness and thickness was evaluated. The tissue stiffness comparison was made using linear regression and the BlandAltman analysis.

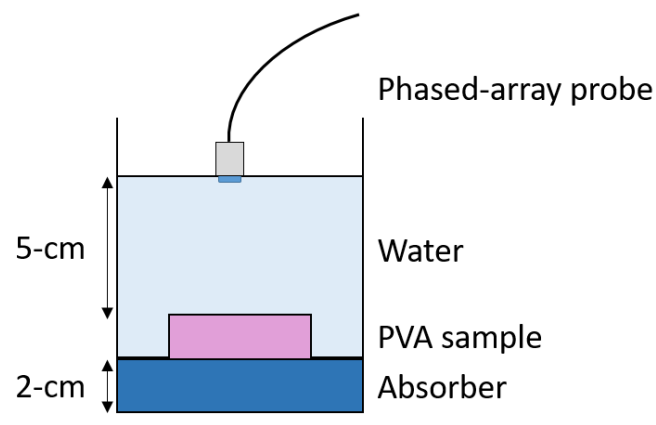

FIGURE 3: THE IN-VITRO EXPERIMENTAL SETUP.

\section{Results}

\section{Evaluation and validation on in-vitro calibrated phantoms}

For the in-vitro acquisitions, shear wave propagation velocities were calculated through the postprocessing analysis as presented in the last section. For each PVA sample, four acquisitions were performed using the commercial SWE mode of the Aixplorer ultrasound scanner system, as gold-standard measurements, and the Myocardial Shear Wave Elastography imaging device. A comparison between the imaging device and the gold-standard was evaluated in terms of shear wave velocity measurements and the corresponding results are presented in Figure 4.

In Figure 4(A), the Myocardial Shear Wave Elastography imaging device measurements as a function of the PVA sample thicknesses are presented with the corresponding averaged and standard-deviation reproducibility values. The corresponding gold-standard measured value, i.e. a shear wave velocity of 3 $\mathrm{m} / \mathrm{s}$, by the Aixplorer ultrasound scanner is also represented. These results showed that the shear velocity decreases when the sample gets thin. For thicknesses inferior to $5-\mathrm{mm}$, the shear wave velocities measured by the MSWEi device present a bias superior to 15-\% when compared to the gold-standard. 
Figures $4(B)$ and $(C)$ show the correlation and the Bland-Altman analysis between MSWEi device and the gold-standard measurements. Results showed a strong correlation between both, corroborated by a Bland-Altman plot. Very good agreement was found for shear velocities less $5 \mathrm{~m} / \mathrm{s}$ than whereas the absolute errors increases at high velocities.

(A)

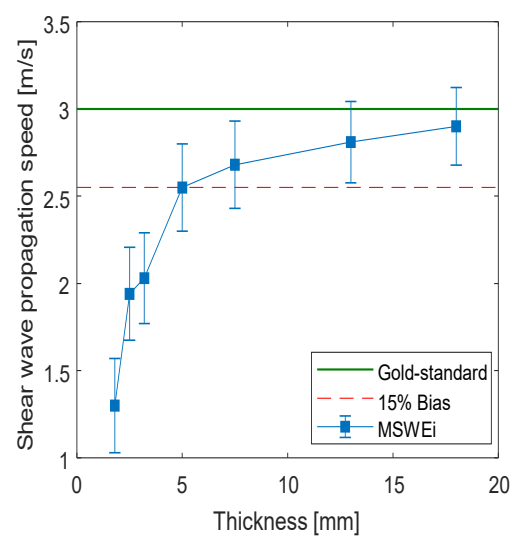

(B)

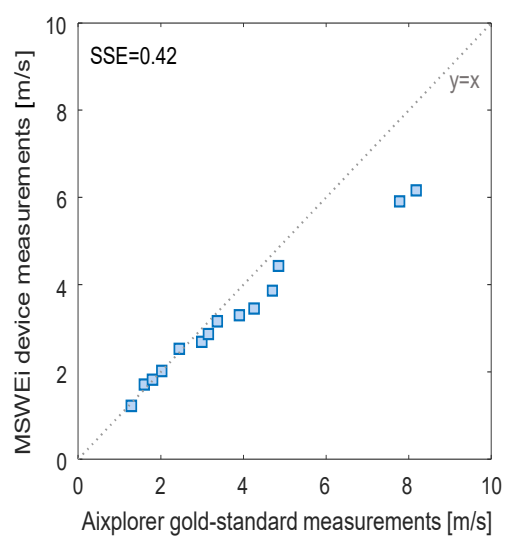

(C)

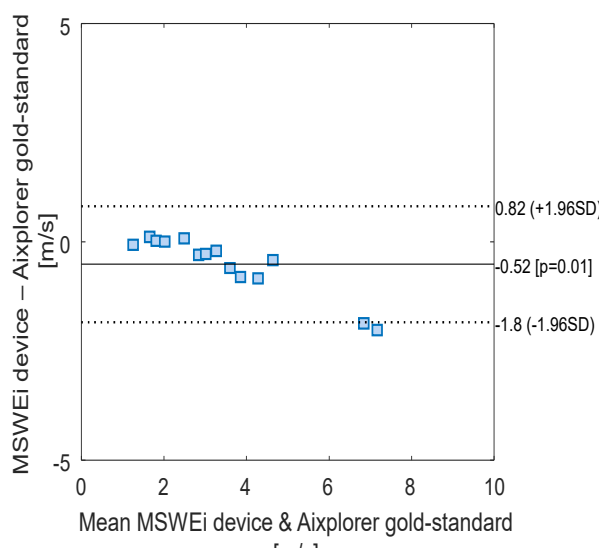

$[\mathrm{m} / \mathrm{s}]$

FIGURE 4: SHEAR WAVE PROPAGATION VELOCITY IN FUNCTION OF THICKNESS AND COMPARISON WITH THE CORRESPONDING GOLD-STANDARD SHEAR WAVE VELOCITY VALUE (A), CORRELATION BETWEEN THE MYOCARDIAL SHEAR WAVE ELASTOGRAPHY IMAGING DEVICE AND THE AIXPLORER GOLD-STANDARD MEASUREMENTS (B), AND BLAND-ALTMAN ANALYSIS OF MYOCARDIAL SHEAR WAVE ELASTOGRAPHY IMAGING DEVICE AND THE AIXPLORER GOLD-STANDARD MEASUREMENTS (C).

\section{Myocardial Shear Wave Elastography imaging device application for clinical purposes}

Figure 5 presents the Myocardial Shear Wave Elastography Imaging device application in clinical practice (Figure 5-A) and an example of an in-vivo transthoracic myocardial shear wave propagation (Figure 5-B). Figure 5(B) shows an example of a possible shear wave propagation tracking in-vivo transthoracically in a long-axis view. When shear wave propagation tracking is possible, the shear wave propagation velocity can be calculated through the post-processing analysis presented.

(A)

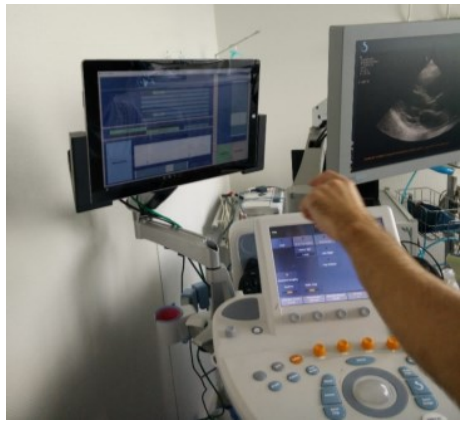

(B)

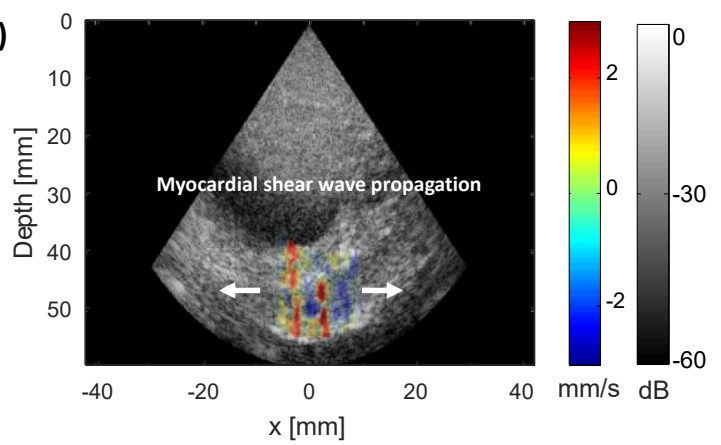

Figure 5: Clinical Myocardial SHEAR WAVE ELASTOGRAPHy IMAGING APPLICATION EXAMPLE. (A) CORRESPONDS TO THE CLINICAL SETUP AND (B) A TISSUE VELOCITY IMAGE OF THE MYOCARDIAL SHEAR WAVE PROPAGATION IN A TRANSTHORACIC LONGAXIS VIEW. 


\section{Discussion}

In this study, we evaluated and validated a new device to perform non-invasive Myocardial Shear Wave Elastography for the purpose of future clinical studies. This prototype consisted on an ultrafast ultrasound scanner, dedicated ultrasound sequences for myocardial shear wave elastography (Papadacci et al. 2014; Correia et al. 2016) and a dedicated graphical user interface that enables the physician to perform the acquisition in a clinical setting. The prototype was evaluated and validated in-vitro using calibrated mimicking tissue phantoms and an example of in-vivo application was also presented.

We tested the performances of the prototype on various calibrated hydrogels of different thickness and stiffness. The shear wave velocity was found to decrease slightly with thickness between 5 and $18-\mathrm{mm}$ and then suddenly dropped for thicknesses inferior to $5 \mathrm{~mm}$. This effect was expected and is mainly due to guided waves propagation when the thickness and the shear wavelength become on the same order of magnitude (Thu-Mai Nguyen et al. 2011). Consequently, the measured shear velocity is underestimated for thickness smaller than $5-\mathrm{mm}$, with a shear wave velocity bias superior to $15 \%$. These results also show that Shear Wave velocity can be accurately measured in tissues with thicknesses superior to 5-mm, with a shear wave velocity bias up to $15 \%$. Therefore, the application of Shear Wave Elastography in clinical practice for myocardial stiffness assessment may be limited on very thin myocardium in specific pathologies, which are characterized by a reduction of the myocardial wall thickness (Mcmurray et al. 2012).

In what concerns the evaluation of different tissues' stiffness and the prototype measurements robustness, the results showed that measurements acquired by MSWEi and the clinical system of the Aixplorer ultrafast ultrasound scanner, i.e. the considered gold-standard, are in good agreement. The Bland-Altman statistical analysis shows that MSWEi measurements are robust up to $5 \mathrm{~m} / \mathrm{s}$ (i.e. a shear modulus of $25 \mathrm{kPa}$ ). For higher shear wave velocities, i.e. stiffer tissues, the MSWEi lacks to track and estimate properly the shear wave propagation velocity. This fact is mainly related to the imaging frame limitation (2000 images/s) due to the large imaging depth required for the heart and imaging quality to track the shear wave propagation. Therefore, the application of the Myocardial Shear Wave Elastography imaging device, as presented, is considered to be limited to a maximum of $5 \mathrm{~m} / \mathrm{s}$, i.e. a shear modulus of $25 \mathrm{kPa}$. We expect this range to be large enough since normal myocardial shear velocity is around $1.5 \mathrm{~m} / \mathrm{s}$ and very stiff pathological myocardium around $4 \mathrm{~m} / \mathrm{s}$ (Villemain et al. n.d.). 
Finally, the application of the prototype was evaluated clinically by trained echocardiographists. The presented results showed that the acquisition can be performed very easily by the physician within a 10 minute examination, but also that myocardial shear wave propagation can be tracked in-vivo, noninvasively and clinically.

\section{Conclusion}

In this study, we presented a novel Myocardial Shear Wave Elastography imaging device to evaluate myocardial stiffness non-invasively using an ultrafast ultrasound scanner in a clinical setting. The imaging setup was evaluated in-vitro and the in-vivo application was also showed. The in-vitro results showed that the Shear Wave Elastography prototype provides accurate and robust measurement in tissue mimicking phantoms for stiffness up to $25 \mathrm{kPa}$.

\section{Acknowledgements}

This work was supported from European Research Council grant under the European Union's Seventh Framework Program (FP/2007-2013) / ERC Grant Agreement n³11025.

\section{Conflict of Interest}

Dr. Tanter is a cofounder of SuperSonic Imagine. All other authors report that they have no relationships relevant to the contents of this paper to disclose.

\section{Bibliography}

Bercoff, J., Tanter, M. \& Fink, M., 2004. Supersonic shear imaging: a new technique for soft tissue elasticity mapping. IEEE Transactions on Ultrasonics, Ferroelectrics and Frequency Control, 51(4), pp.396-409. Available at: http://ieeexplore.ieee.org/articleDetails.jsp?arnumber=1320804 [Accessed March 29, 2014].

Bonnefous, O., Pesque, P. \& Bernard, X., 1986. A new velocity estimator for color flow mapping. In Proc. IEEE Ultrason. Symp. IEEE, pp. 855-860.

Bouchard, R.R. et al., 2011. Acoustic radiation force-driven assessment of myocardial elasticity using the displacement ratio rate (DRR) method. Ultrasound in medicine \& biology, 37(7), pp.1087-100. Available at: http://www.sciencedirect.com/science/article/pii/S030156291100202X [Accessed August 21, 2014].

Correia, M. et al., 2016. Ultrafast Harmonic Coherent Compound (UHCC) imaging for high frame rate echocardiography and Shear Wave Elastography. IEEE Transactions on Ultrasonics, Ferroelectrics, and Frequency Control, pp.1-1. Available at: http://ieeexplore.ieee.org/lpdocs/epic03/wrapper.htm?arnumber=7407422.

Gennisson, J.-L. et al., 2013. Ultrasound elastography: principles and techniques. Diagnostic and interventional imaging, 94(5), pp.487-95. Available at: http://www.ncbi.nlm.nih.gov/pubmed/23619292 [Accessed September 9, 2014]. 
Hsu, S.J. et al., 2007. In vivo assessment of myocardial stiffness with acoustic radiation force impulse imaging. Ultrasound in medicine \& biology, 33(11), pp.1706-19. Available at: http://www.sciencedirect.com/science/article/pii/S0301562907002530 [Accessed August 13, 2014].

McLaughlin, J. \& Renzi, D., 2006. Using level set based inversion of arrival times to recover shear wave speed in transient elastography and supersonic imaging. Inverse Problems, 22(2), pp.707-725.

Mcmurray, J.J. V et al., 2012. ESC Guidelines for the diagnosis and treatment of acute and chronic heart failure 2012. European Journal of Heart Failure, 14(8), pp.803-869.

Papadacci, C. et al., 2014. High-contrast ultrafast imaging of the heart. IEEE transactions on ultrasonics, ferroelectrics, and frequency control, 61(2), pp.288-301. Available at: http://www.ncbi.nlm.nih.gov/pubmed/24474135 [Accessed April 15, 2014].

Pernot, M. et al., 2011. Real-time assessment of myocardial contractility using shear wave imaging. Journal of the American College of Cardiology, 58(1), pp.65-72. Available at: http://www.ncbi.nlm.nih.gov/pubmed/21700091 [Accessed April 11, 2014].

Pernot, M. et al., 2016. Shear Wave Imaging of Passive Diastolic Myocardial Stiffness: Stunned Versus Infarcted Myocardium. JACC. Cardiovascular imaging. Available at: http://www.sciencedirect.com/science/article/pii/S1936878X16302273 [Accessed May 31, 2016].

Pislaru, C. et al., 2009. Shearwave dispersion ultrasound vibrometry applied to in vivo myocardium. Conference proceedings :... Annual International Conference of the IEEE Engineering in Medicine and Biology Society. IEEE Engineering in Medicine and Biology Society. Conference, 2009, pp.28914. Available at: http://www.ncbi.nlm.nih.gov/pubmed/19964051 [Accessed August 21, 2014].

Sandrin, L. et al., 1999. Time-Resolved Pulsed Elastography with Ultrafast Ultrasonic Imaging. Ultrasonic Imaging, 21(4), pp.259-272. Available at: http://uix.sagepub.com/content/21/4/259.abstract [Accessed October 24, 2014].

Song, P. et al., 2013. Improved Shear Wave Motion Detection Using Pulse-Inversion Harmonic Imaging with a Phased Array Transducer. IEEE transactions on medical imaging, (c). Available at: http://www.ncbi.nlm.nih.gov/pubmed/24021638.

Thu-Mai Nguyen et al., 2011. Assessment of viscous and elastic properties of sub-wavelength layered soft tissues using shear wave spectroscopy: Theoretical framework and in vitro experimental validation. IEEE Transactions on Ultrasonics, Ferroelectrics and Frequency Control, 58(11), pp.23052315. Available at: http://ieeexplore.ieee.org/document/6071049/ [Accessed May 26, 2017].

Villemain, O. et al., Myocardial stiffness assessment using shear wave imaging in pediatric hypertrophic cardiomyopathy. 